\title{
Un recorrido por territorios en conflicto*
}

\author{
Darcy Tetreault \\ Universidad Autónoma de Zacatecas
}

$\mathrm{E}$

1 libro Territorios en conflicto, coordinado por Jorge Federico Eufracio Jaramillo y publicado por El Colegio de Jalisco, reúne siete ensayos acerca de disputas socioambientales en México, con estudios de caso en el Occidente y Centro Norte del país. El eje aglutinador es la problematización del territorio. El objetivo es, en palabras del coordinador: "generar conocimiento o ensayar enfoques diferentes a partir de una revalorización de la variable territorial [...] como una compleja y conflictiva red de interconexiones, un fluido o un tejido, una realidad relacional" (p. 21). De acuerdo con esto, los capítulos incluidos en este libro ponen en el centro del análisis las relaciones de poder entre distintos grupos y actores sociales. Toman en cuenta no solamente los aspectos fisco-materiales de los territorios en conflicto, sino también la construcción simbólica e histórica de la territorialidad.

El capítulo introductorio se titula "El territorio y sus debates: sobre sus cualidades analíticas y dimensiones multidisciplinares". Aquí, el coordinador explica el origen de la obra y su objetivo general, para luego explorar el concepto de territorio y señalar algunos traslapes y matices con los conceptos de "espacio" " "región". Señala cómo estos conceptos tienen raíces en la geografía del estado, la cual representa "una territorialización desde arriba" (p. 11). Nuestro autor apunta hacia un nuevo paradigma que pretende superar las nociones de "espacio contenedor" y "espacio inerte". Parafraseando a Milton Santos, afirma que el espacio "no está formado únicamente por las cosas [...] sino también por la sociedad misma" (p. 14). De esta manera, argumenta que el espacio es "eminentemente social" debido a "la inseparabilidad entre sistemas de objetos y de acciones" (p. 14). Por tanto, aboga por una epistemología que se enfoque en relaciones de poder entre diferentes grupos, actores y clases sociales. Este primer capítulo aporta un marco conceptual para la obra, además de presentar un resumen capitular.

En el segundo capítulo, titulado "Relaciones regionales: uso y gestión", el autor, Carlos Riojas, de la Universidad de Guadalajara, propone el objetivo de "exponer una variedad de relaciones regionales que se han gestado a lo largo del tiempo", en un esfuerzo por poner de relieve las articulaciones entre lo que él llama factores internos y externos, o endógenos y exógenos. Tiene dos secciones: la primera

Reseña del libro de Jorge Federico Eufracio Jaramillo (coord.), Territorios en conflicto. Ensayos acerca de disputas socioambientales, Zapopan, El Colegio de Jalisco, 2016. 
esboza una perspectiva de las vinculaciones entre los factores internos y externos; la segunda hace un breve recorrido histórico para analizar algunas articulaciones específicas en el caso de México, particularmente en lo que concierne el uso y la gestión del agua y los bosques.

Carlos Riojas recurre a diversas experiencias de casos a nivel internacional para ilustrar "las peculiaridades inherentes al funcionamiento regional" (p.31). Ubica los factores externos a nivel global, con referencia al proyecto y la ideología neoliberales, cuyas recetas económicas desatan fuerzas homogeneizadoras que enfrentan "una amplia diversidad de escenarios regionales" (p. 35). Para ilustrar esto, el texto nos lleva por un recorrido histórico desde tiempos prehispánicos hasta el presente, apuntando a las relaciones de poder imbricadas en la formación, apropiación y transformación de los territorios; y tomando en cuenta el impacto de introducir nuevas tecnologías, la construcción de acueductos y grandes represas para la irrigación, el desarrollo de sistemas de comunicación y la creación de áreas de conservación, entre otros factores. Todo esto gira en torno al manejo de recursos hídricos y forestales en México, con algunas desviaciones.

En este análisis, el autor observa tendencias centralizadoras en torno del Estado, sobre todo a partir del porfiriato, cuando el control administrativo sobre los recursos naturales de la nación empezó a pasar de los hacendados y los gobiernos locales al gobierno federal; una tendencia que fue reforzada después de la Revolución Mexicana hasta los años ochenta del siglo pasado. En sentido contrario, observa a partir de la crisis de la deuda de 1982 una transnacionalización de las iniciativas vinculadas a los recursos naturales, y pone el ejemplo de los proyectos mineros llevados a cabo por empresas multinacionales. Al final, llega a la conclusión de que, a pesar de la persistencia de tendencias centralizadoras y homogeneizadoras, "existen características regionales que confirman más bien la heterogeneidad" (p. 45). Con todo, el autor alcanza altos niveles de abstracción y generalización, de acuerdo con una perspectiva de larga duración y amplio alcance geográfico.

El tercer capítulo se titula "El corazón del universo en disputa. Un estudio de caso del conflicto por la territorialización de un sitio sagrado natural de México". Su autora es María Julieta Lamberti, quien estudió el doctorado en El Colegio de México bajo la dirección de Rodolfo Stavenhagen. El estudio de caso al que se refiere en el título es Wirikuta, el territorio sagrado de los wixaritari que se ubica en el estado de San Luis Potosí. En el mismo territorio se encuentra Real de Catorce, un pueblo turístico con una historia minera alrededor del cual se han detectado reservas de plata que pueden ser explotadas de manera rentable con las tecnologías existentes. Una compañía canadiense -que la autora llama Production Silver, un seudónimo para First Majestic Silver- busca el camino político para explotar los minerales del subsuelo. 
La autora empieza el capítulo situando el estudio de caso en el entrecruzamiento de dos procesos: el avance del extractivismo minero durante la primera década del siglo xxi y el resurgimiento de la cuestión indígena. Su marco analítico-conceptual se nutre de tres enfoques: la teoría del actor-red; los estudios de la ciencia, tecnología y sociedad, y las investigaciones sobre la política indígena. De esta manera, conceptualiza los territorios "como ensamblajes particulares de objetos, espacios, narrativas, poderes, personas y diosas, que son construidos por colectivos híbridos" (p. 52). Estos colectivos híbridos son los actores que operan en redes.

La autora describe las estrategias de territorialización del "colectivo antiminero en Wirikuta" y las contrapone a las del colectivo minero y el colectivo gubernamental. Nos explica que las estrategias del primero incluyen: demostrar la centralidad de Wirikuta en la cosmología de los wixaritari; judicializar la ocupación tradicional del territorio; enfatizar la integralidad del territorio, y abogar por el reconocimiento de Wirikuta como patrimonio cultural y natural de la humanidad ante la Organización de las Naciones Unidas para la Educación, la Ciencia y la Cultura (Unesco). Estas estrategias, nos explica, chocan con las del colectivo minero, que ha proyectado una territorialidad basada en un discurso que enfatiza la historia de las actividades mineras en la zona y asevera que esta, por sus características geológicas, tiene una "vocación minera". Vale la pena mencionar que, además de Production Silver, el colectivo minero descrito por la autora incluye agencias gubernamentales como el Servicio Geológico Mexicano, que refuerza este discurso.

También hay agencias gubernamentales cuya razón de ser es legitimar el Estado neoliberal al promover programas sociales y de protección ambiental. Nuestra autora describe los instrumentos utilizados por un colectivo formado por algunas de ellas para construir una "territorialidad gubernamental". Estos instrumentos incluyen: la delimitación de un polígono que abarca los sitios sagrados del pueblo wixarika; el plan de ordenamiento territorial y ecológico de San Luis Potosí, y el fallido decreto de una reserva de la biósfera. En la conclusión, afirma que "existen múltiples formas de territorializar un espacio" y que, por tanto, la conceptualización del territorio debe ser "fluida" (p. 70). Cabe hacer notar que la información presentada en este capítulo refleja una convivencia cercana con el pueblo wixarika.

El cuarto capítulo, escrito por Abril Fabiola Casas Cervantes, egresada de la Maestría Interinstitucional en Políticas Públicas de El Colegio de Jalisco, se titula "La gestión comunitaria y los conflictos por el agua en el manantial de Patamburapio, Michoacán, 2009-2014". El estudio de caso trata del manejo colectivo del agua que abastece cuatro localidades de los municipios de Tocumbo y Tingüindín; analiza los retos que han enfrentado los comités de agua de las cuatro localidades desde 2009, cuando se agudizaron los problemas de escasez del líquido. Su objetivo es exponer la situación de conflicto en torno a dicho manantial, al describir el papel de los actores involucrados, principalmente los comités del agua, las autoridades 
municipales y los agricultores locales. En cuanto a la Comisión Nacional del Agua (Conagua), la autora destaca su ausencia, ya que dicha instancia ha ignorado las peticiones de los comités de agua para que regule las tomas agrícolas.

El capítulo tiene siete secciones, incluyendo la introducción y la conclusión. En la segunda, la autora coincide con Elinor Ostrom en cuanto a ver los cuerpos de agua como un recurso de uso común. Este acercamiento, a propósito, ha sido criticado por Gustavo Esteva, quien lo considera demasiado economicista; y también por los autores del libro Común, Christian Laval y Pierre Dardot, quienes advierten en contra de buscar lo común en las propiedades intrínsecas de ciertos elementos naturales, como el agua. En todo caso, el diálogo con Ostrom le permite a nuestra autora abordar una discusión teórico-conceptual sobre la gestión comunitaria del agua y los importantes retos que esta enfrenta en la práctica.

La tercera sección explora y define el concepto de conflicto con respecto al agua; en ella se hace notar que "las relaciones de poder juegan un papel fundamental en la comprensión" del conflicto (p. 81). La cuarta hace una descripción detallada de la región abastecida por el manantial; y la quinta describe el funcionamiento de los comités de agua. La sexta sección es clave, puesto que analiza el conflicto en torno al agua a partir de las causas de la escasez; entre las cuales destaca el consumo de grandes cantidades de agua por los agricultores, incluso con tomas clandestinas, sin regulación por parte de la Conagua ni por las autoridades locales. Según los testimonios de los entrevistados, los agricultores involucrados se niegan a comunicarse con los comités y la presencia de grupos delictivos complica la situación. Por tanto, nuestra investigadora concluye que "los comités del agua están atados de manos" (p. 97), dejando así un pronóstico bastante pesimista. Con todo, el estudio de caso, tal como se presenta, ayuda a aterrizar los debates sobre la autonomía al dimensionar algunos de los retos que las luchas por lo común tienen que enfrentar en la práctica, apuntando a la necesidad de lidiar de una manera u otra con el Estado.

El quinto capítulo fue escrito por Anahí Copitzy Gómez Fuentes, profesora investigadora de El Colegio de Jalisco, y se titula "Cronología y análisis del conflicto por la construcción de la presa El Zapotillo, 2005 a 2014”. La autora recurre a una revisión sistemática de notas publicadas en los diarios La Jornada Jalisco y Mural para reconstruir el proceso de oposición social a la presa El Zapotillo. Por otra parte, este ejercicio se sostiene sobre la base de varios años de investigación de campo y una revisión de la literatura relevante. Para organizar su análisis, Anahí utiliza una periodización que coincide con los sexenios del gobierno estatal; empieza en 2005, cuando se anunció el proyecto de construir la presa. De esta manera, el primer período abarca los últimos dos años del gobierno de Francisco Ramírez Acuña (2005 y 2006), el segundo corresponde al sexenio de Emilio González Márquez (20072012) y el tercero, a los primeros dos años del gobierno de Aristóteles Sandoval Díaz (2013 y 2014). En las conclusiones, Anahí destaca "la desinformación sistemá- 
tica hacia los afectados" (p. 122) y califica como "poco eficientes" a las estrategias de la Conagua y la Comisión Estatal de Agua (p. 123). Finalmente, enumera una serie de factores que han incidido en el conflicto, para guiar investigaciones posteriores.

El título del sexto capítulo es "El caso de Arcediano y el abasto de agua: un conflicto socioambiental que desapareció un pueblo", y fue escrito por Ivonne Álvarez Gutiérrez, doctorante en el programa de ciencias sociales de El Colegio de Jalisco. Analiza el conflicto socioambiental en torno a los planes para construir la presa Arcediano en la Barranca de Huentitán, con el propósito de abastecer la zona metropolitana de Guadalajara de agua potable. Como hace notar la autora, estos planes provocaron un conflicto no solo por el desplazamiento de los pobladores de la Barranca, sino también por los riesgos asociados con los altos niveles de contaminación del agua que se pretendía usar para el consumo humano. El texto se divide en cuatro apartados, aunque en la introducción solo se mencionan tres. El primero esboza los postulados de la ecología política como corriente teórico-metodológica para analizar los conflictos socioambientales. El segundo describe el proyecto Arcediano en términos técnicos y analiza el proceso político para impulsar su realización. El tercero esboza las inconsistencias del proyecto, entre las cuales destaca la ubicación de la presa en un área de conservación, además de la pésima calidad del agua del río Santiago. El siguiente apartado identifica los actores involucrados en la defensa de la Barranca de Huentitán y analiza las estrategias legales empleadas por ellos. En las conclusiones, la autora contrasta las distintas valorizaciones del territorio en conflicto y retoma la afirmación de que tienen dos dimensiones: una material y otra simbólica.

El séptimo y último capítulo del libro también trata de la Barranca de Huentitán, pero desde otro enfoque. Se titula "Paisaje y territorio: la Barranca de Huentitán en un recorrido geohistórico". El autor, Christian Grimaldo, doctorante del programa de cIESAS-Occidente, plantea en la introducción un acercamiento que parte de dos elementos básicos: el tiempo y el espacio. Este acercamiento pretende ir mucho más allá de simplemente "enunciar el nombre de un lugar y unas fechas precisas" (p. 157), al abordar una "descripción geohistórica" del "surgimiento, desaparición y encuentro de actores, grupos e instituciones" vinculados al paisaje en cuestión (p. 158). El objetivo del autor es demostrar la relevancia analítica del análisis geohistórico, con un estudio de caso sobre la Barranca de Huentitán.

Así, después de localizar y caracterizar la Barranca, y de definir algunos conceptos clave como paisaje, territorio y apropiación, empieza el recorrido desde tiempos prehispánicos. Presta mucho atención a la etapa de los arrieros, quienes fueron, en sus palabras, "el principal medio de transporte de mercancías entre ciudades y campo durante por lo menos tres siglos" (p. 165); y explica por qué este medio de transporte comercial se utilizó en rutas que pasaron por Huentitán hasta la década de los años cincuenta del siglo pasado. Asimismo, en las siguientes secciones ana- 
liza las múltiples transformaciones de la Barranca impulsadas por los procesos de industrialización, incluyendo por ejemplo la construcción de una planta hidroeléctrica a finales del siglo XIX, y luego, a partir de los años setenta del siglo pasado, la contaminación del río Santiago.

No aborda los conflictos en torno a esta contaminación. Asimismo, el conflicto en torno a la presa Arcediano está en el trasfondo. El enfoque, más bien -en la parte que trata de la etapa contemporánea- es sobre las tensiones que existen entre los usuarios del paisaje de la Barranca. Por un lado, "los tradicionales", quienes tienen años haciendo ejercicio allí y han formado lazos de amistad entre sí; y por el mismo lado, los ex habitantes de las localidades de Las Juntas y Arcediano, quienes fueron desplazados por los planes para construir la presa Arcediano; y por el otro lado, "los nuevos visitantes", quienes no respetan las reglas informales de los otros dos grupos al hacer ruido, dejar atrás los excrementos de sus mascotas, alcoholizarse, etc. Al final del recorrido, nuestro investigador retoma las "oposiciones entre usos y representaciones del territorio" que corresponde a la Barranca de Huentitán (p. 175); reconoce las conexiones entre las transformaciones de este paisaje y el sistema económico mundial; y también enfatiza las dos dimensiones de los conflictos territoriales: la utilitario-funcional y la simbólico-cultural.

Con todo, el libro constituye una espléndida colección de ensayos sobre los territorios mexicanos en conflicto. Siete ensayos que exhiben acercamientos innovadores, diversas escalas de análisis y estudios de caso en el ámbito local. Combina los trabajos de investigadores experimentados y reconocidos con los de algunos estudiantes destacados de los programas de posgrado de El Colegio de Jalisco y CIESAS-Occidente. La problematización del territorio en torno a los conflictos socioambientales constituye el denominador común, así como el reconocimiento y la exploración de las dimensiones material y simbólica de estos conflictos.

Tengo algunas observaciones críticas de segundo orden. Por ejemplo, en el capítulo sobre Wirikuta, ¿por qué usar seudónimos para un caso tan conocido a nivel nacional e internacional? Otro ejemplo: en el capítulo sobre el manejo comunitario del agua en Michoacán, me quedé preguntando al final de la lectura sobre la etnicidad de los actores locales y si el sistema de manejo comunitario tiene raíces en instituciones de autogobierno indígena. Considerando la obra en su totalidad, me llamó la atención que los autores no citan algunos estudiosos reconocidos sobre el tema; por ejemplo, Víctor Toledo y Mina Navarro, cuyos trabajos sobre los conflictos socioambientales en México ponen énfasis en cuestiones territoriales. Y, finalmente, creo que hubiera sido enriquecedor incluir al final del libro un capítulo para presentar algunas conclusiones globales.

No obstante estas críticas, considero que el libro hace contribuciones significativas a los debates sobre la conflictividad socioambiental en México. Amplía nuestros conocimientos sobre casos específicos y tendencias generales; y, llama a prestar 
atención a la centralidad del territorio y la territorialidad. Sale a luz en horabuena, en el contexto de una creciente crisis civilizatoria multidimensional, cuya salida -creo yo- será definida en gran medida por las luchas en torno a los usos y representaciones ecoterritoriales. 